\title{
Sudden asystole during radiofrequency ablation: a case report and literature review
}

\author{
He-Sheng Hu, Mei Xue, Rui Xu, Xiao-jun Wang, Ming-you Chen and Su-Hua Yan*
}

\begin{abstract}
Background: Radiofrequency (RF) ablation is a widely accepted and ideal therapeutic tool to cure some tachycardias. The occurrence of complications varies depending on the procedure being performed. Sudden unexpected prolonged asystole is rare for most ablation procedures and the underlying mechanisms remain unclear.

Case presentation: A case of sudden prolonged asystole induced by RF ablation of a concealed left free wall accessory in a 59-year-old woman with recurrent tachycardia. RF application provoked progressive slowing of the sinus rhythm and then a 13.2-second period of asystole ensued. Asystole was self-healing and no complications were seen in the following follow-up.

Conclusions: RF ablation may develop prolonged asystole due to vagus response caused by stimulation of unmyelinated vagal C-fibers or ganglionated plexus (GP). Reflexible asystole is reproducible and resolves independently, without affecting the procedure of RF ablation.
\end{abstract}

Keywords: Radiofrequency ablation, Asystole, Vagal response, Ganglionated plexus

\section{Background}

Radiofrequency (RF) ablation has revolutionized treatment of arrhythmias and is now considered to be a firstline therapy for some tachycardias [1]. RF is curative, long-term medication is not required. Its occurrence of complications varies depending on the procedure being performed, and serious complications are rare for most ablation procedure [2]. Bradycardia with hypotension is common during ablation procedures, chiefly due to cardiac reflexes stimulated by chemoreceptors or mechanoreceptors found throughout the vessels, atrium, ventricle, pericardium, and coronary arteries. This cardio-inhibitory reflex can increase parasympathetic tone and cause bradycardia, hypotension, and even cardiac asystole. To the best of our knowledge, sudden unexpected asystole has been rare in the past three decades, spanning the time since the first report of successful RF ablation of tachyarrhythmia. However, the actual incidence of asystole due to RF is unknown.

Here, we present a case of prolonged asystolic episode induced by RF ablation of a concealed left free wall

\footnotetext{
* Correspondence: yansuhua5537@163.com

Department of Cardiology, Shandong Provincial Qianfoshan Hospital, Shandong University, 250014 Jinan, PR China
}

accessory pathway through a transaortic approach. We also briefly review previously reported cases to explore the mechanism of sudden asystole and its clinical significance.

\section{Case presentation}

A 59-year-old female patient presented with complaints of recurrent palpitations over the past three years. Paroxysmal supraventricular tachycardia (SVT) was diagnosed on several emergency department visits. A 12-lead surface electrogram and physical examination were normal. The SVT had a narrow QRS-complex during the attacks $(230 \mathrm{bpm})$. After informed consent was obtained, the patient received an electrophysiologic study (EPS) and catheter ablation. Multipolar electrode catheters (Biosense Webster, USA) were positioned in the His-bundle region, right ventricular apex, and coronary sinus (CS). Prior to EPS, blood pressure was $121 / 67 \mathrm{~mm} \mathrm{Hg}$ and pulse was $72 \mathrm{bpm}$. Programmed atrial stimulation indicated that sinus and atrioventricular (AV) nodal functions were normal. $\mathrm{CS}_{1,2}$ proceeded during ventricular extrastimulus testing and a basic drive $\left(\mathrm{S}_{1} \mathrm{~S}_{1}\right)$ cycle length of $400 \mathrm{~ms}$ of ventricular pacing induced AV re-entrant tachycardia (AVRT). The ventriculoatrial (VA) conduction during ventricular 
pacing was consistent with inducible AVRT. A concealed left free wall accessory pathway was confirmed and selected for mapping and ablation via a transaortic approach. The target site was obtained above the mitral annulus during AVRT and RF energy was delivered $\left(60^{\circ} \mathrm{C}\right.$; $30 \mathrm{~W}$ ) using a temperature-controlled catheter (Biosense Webster). Tachycardia terminated after a 2-sec ablation and ablation was continued for $25 \mathrm{sec}$ more. At $22 \mathrm{sec}$ after the onset of energy delivery, sudden cardiac asystole lasted for $13.2 \mathrm{sec}$ followed by profound sinus rate slowing though ablation was aborted 2 sec after asystole emerged (Figure 1). No escape rhythm emerged. Moreover, AV interval was not changed before and after asystole. After sinus rhythm was rescued, blood pressure was 165/97 mm $\mathrm{Hg}$ and ventricular pacing indicated VA disassociation. Multiple ventricular pacing and programmed electrical stimuli for a subsequent $30 \mathrm{~min}$ revealed VA disassociation and no induction of tachycardia. Sinus node function AV conduction was normal. The patient no longer experienced tachycardia and syncope 9 months post-ablation.

\section{Discussion}

Here, we describe a rare complication during RF ablation above the mitral annulus during a retrograde aortic approach to ablate a left free wall accessory pathway, characterized by prolonged asystole which resolved on its own. Prolonged asystole has been reported to occur not only occurred during SVT ablation [3-7], but atrial fibrillation ablation [8-10].

\section{Clinical characteristics}

Based on prior case reports and this case, prolonged asystole induced by ablation can be described as depicted in Table 1. These have occurred in individuals with no apparent structural heart disease. Prolonged asystole can occur during slow pathway ablation, at the pulmonary vein, the intra-coronary sinus and around the mitral annulus. Asystole is prone to be reproducible under ablation procedures and Table 1 depicts the duration of each asystolic event (mean $=9.6 \pm 3.9 \mathrm{sec}$ ) as well as other symptoms patients experienced seemed to resolve on its own and not affect success of ablation. Asystole that does not influence the ablation procedure but may require pacing or atropine. Among the current 10 cases, only one patient failed because the patient requested to complete the process due to anxiety after the second asystole [7].

\section{Mechanism}

Cardiac asystole has been observed during ablation of various arrhythmias and some intra-cardiac procedures and no clear mechanistic explanation for these events is available. A Bezold-Jarisch-like reflex was a putative mechanism because this is thought to be a cardiovascular decompressor reflex originating in sensory receptors with vagal efferent discharge to the heart [11]. RF current delivery may acutely stimulate the unmyelinated vagal $\mathrm{C}$-fibers which are predominantly distributed within the right atrium lateral wall, the left atrium roof, around the four pulmonary veins, and in the posterior left ventricle. These fibers reflexively increase parasympathetic tone, decrease sympathetic activity, mediate bradycardia-hypotension, and then cause prolonged asystole.

However, bradycardia only lasts 1 to $2 \mathrm{sec}$ after cessation of direct efferent vagal fiber stimulation [12] which is unlike prolonged asystole which persisted for up to $3 \mathrm{sec}$ after cessation of RF current in the series case. Another potential mechanism is the ganglionated plexus (GP) response. GPs embed within epicardial fat pads and the ligament of Marshall, receiving input from the extrinsic cardiac nervous system. This response includes

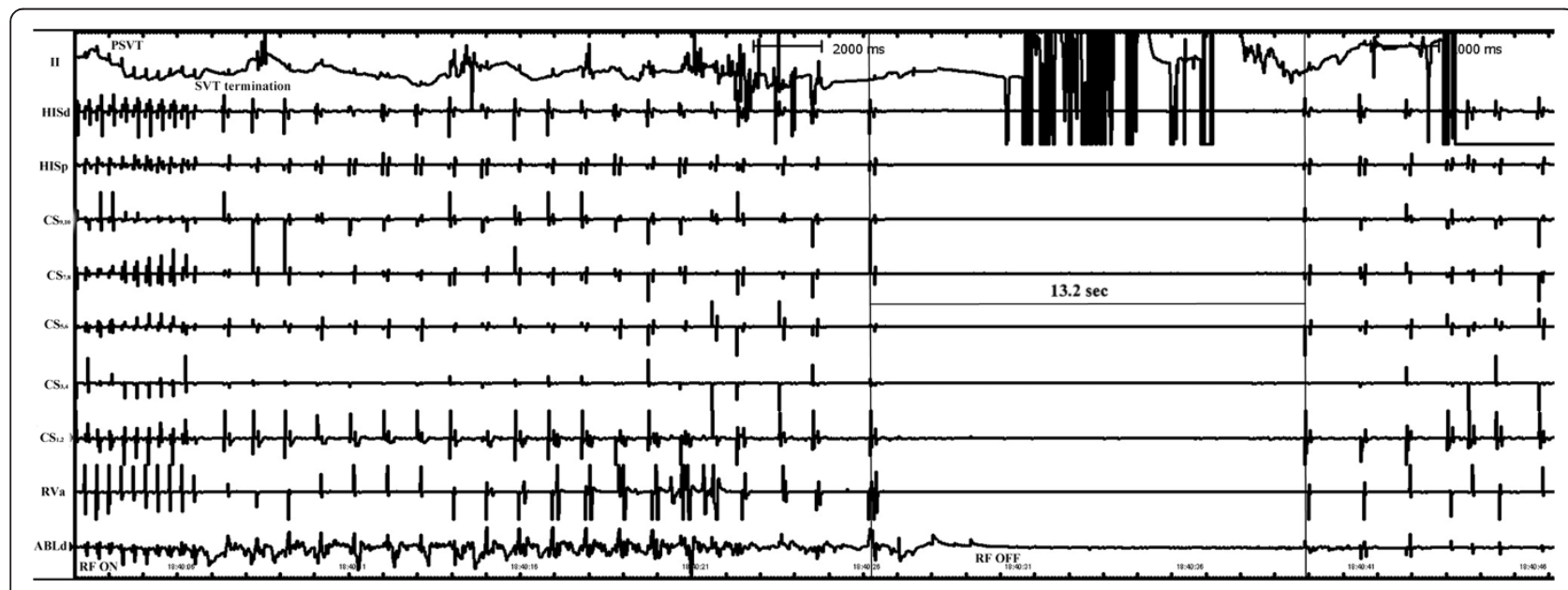

Figure 1 ECG (lead II) and intracardiac electrogram showed an asystole of $13.2 \mathrm{~s}$ during radiofrequency energy applications at the atrial aspect of the mitral annulus for the left anterolateral accessory pathway. 
Table 1 Features of 10 Cases of Prolonged Asystole induced by radiofrequency ablation

\begin{tabular}{|c|c|c|c|c|c|c|c|c|c|}
\hline Ref. number & Age(yrs)/sex & Diagnosis & Ablation site & Asystole time(s) & Repeated asystole & Therapy & Accompanying syndrome & Result & Adjacent ganglionated plexus \\
\hline [8] & $45 / F$ & PAF & LSPV & 8.2 & No mention & Ventricular pacing & No mention & S & LSAGP \\
\hline [8] & $80 / \mathrm{M}$ & PAF & LSPV & 9.5 & No mention & Ventricular pacing & No mention & S & LSAGP \\
\hline [9] & $54 / F$ & PAF & LSPV & 17 & No mention & None & Syncope & S & LSAGP \\
\hline [3] & $35 / M$ & AVRT & Coronary sinus & 8 & Y & None & Near fainting; slight pain & S & PLLAGP \\
\hline [4] & $28 / F$ & AVRT & LVFW & 5.5 & Y & Ventricular pacing & Near syncope; mild pain & S & PLLAGP \\
\hline [5] & $69 / F$ & AVNRT & $\mathrm{SP}$ & 10.3 & Y & Atropine & No discomfort & S & PMLAGP \\
\hline [6] & $52 / F$ & AVNRT & $\mathrm{SP}$ & 4.6 & Y & None & No pain & S & PMLAGP \\
\hline [7] & $43 / \mathrm{M}$ & AVNRT & SP & 6.9 & Y & None & Mild chest pain & $\mathrm{F}$ & PMLAGP \\
\hline [10] & $67 / M$ & PAF & LSPV & 12.5 & Y & Ventricular pacing & No mention & S & LSAGP \\
\hline Present & $59 / F$ & AVRT & LVFW & 13.2 & $\mathrm{~N}$ & None & Syncope & S & PLLAGP \\
\hline
\end{tabular}

Abbreviations: AVNRT atrioventricular nodal reentrant tachycardia, AVRT atrioventricular reentrant tachycardia, PAF paroxysmal atrial fibrillation, SP slow pathway, $L V F W$ left ventricular free wall, $L S P V$ left superior pulmonary, $Y$ Yes, $N$ No, $S$ Success, $F$ Failure, LSAGP Left superior atrial ganglionated plexus, PMLAGP posteromedial left atrial ganglionated plexus, PLLAGP posterolateral left atrial ganglionated plexus. 
afferent neurons, post-ganglionic efferent parasympathetic and sympathetic neurons, and numerous interconnecting neurons that provide communication within and between the GPs [13]. Capulzini and co-workers reported that targeting the area corresponding to the left superior GP provoked induction of atrial fibrillation and a 12.5-sec asystole [9]. Mathuria and his colleagues speculated that attempted slow-pathway ablation might stimulate nearby GPs and induce cardiac asystole [6]. Whether the GP response is a plausible mechanism that causes reflexive cardio-inhibition is uncertain, evidences suggest that prolonged asystole can be induced by direct GP stimulation. First, targeted GP high-frequency stimulation (HFS) produced a notable vagal response including prolonged asystole. GP ablation has been found to be effective in the treatment of AF and HFS is commonly used to verify localization and ablation of GP via evoked vagal reflexes [14]. HFS of GPs elicited $3.1 \mathrm{sec}$ ventricular asystole via an endocardial approach [15] and a $7.85 \mathrm{sec}$ AV block in the left atrial epicardium prior to RF ablation in patients with AF [16]. Secondly, GPs are composed of sympathetic and parasympathetic nerves, the majority of which are cholinergic [17]. Stimulation of GPs predominantly elicited more vagal effects, as seen in targeted GP ablation. Thirdly, ablation sites are close to the GP area (Table 1). Slow pathway ablation most likely corresponded with the interatrial septal GP formed by the two posterior atrial GPs that are fused and extend anteriorly into the interatrial septum. Ablation of the coronary sinus and left superior pulmonary (LSPV) occurs in areas adjacent to the posterolateral left atrial GP and the superior left atrial GP respectively. Moreover, asystole may occur more commonly in the left atrium and the interatrial septum, which has abundant GPs $(60 \%, 3 / 5)$. Tsai et al. [8] reported that $15 \%(6 / 40)$ of patients developed bradycardia-hypotension syndrome during energy delivery to the superior pulmonary veins for treatment of paroxysmal atrial fibrillation. The present case may be caused by stimulation of GP or its adjacent area which facilitated sudden cardiac asystole during attempted ablation in the region of left free wall accessory pathway. Moreover, pain and other factors (nausea, sedation and anxiety) may also be associated with the response. In these collected cases, 3 patients $(3 / 10,30 \%)$ showed chest pain. Clinically, RF induced pain was common and slight. And asystole still emerged repeatedly though the patient was familiar with the procedure and pain [3]. The causal mechanism is sometimes implicated in clinical situations and study should be furthered to disclose the intrinsic mechanism.

\section{Conclusions}

However, profound sinus rate slowing and no AV interval changing during asystole may imply preferential sinus node effects. The intrinsic cardiac nervous system is very complex and its precise anatomy has not been explored in detail. The above mentioned causal mechanisms can only be speculative. The exact nature involved is not unclear. RF ablation may develop prolonged asystole due to vagus response caused by stimulation of unmyelinated vagal $\mathrm{C}$-fibers or GP. Although prior case reports have shown the reflexible asystole may not affect RF ablation procedures, clinical cardiologists should be aware of this rare complication during RF ablation and properly manage these events, especially ablating the GP and its adjacent area. If asystole recurs frequently, ventricular pacing and atropine (and similar compounds) may be helpful for performing RF ablation procedure.

\section{Consent}

Written informed consent was obtained from the patient for publication of this case report and accompanying images. A copy of the written consent is available for review by the Editor-in-Chief of this journal.

\section{Abbreviations}

AVNRT: Atrioventricular nodal reentrant tachycardia; AVRT: Atrioventricular reentrant tachycardia; CS: Coronary sinus; EPS: Electrophysiologic study; F: Failure; GP: Ganglionated plexus; LSAGP: Left superior atrial ganglionated plexus; LSPV: Left superior pulmonary; LVFW: Left ventricular free wall; RF: Adiofrequency; N: No; PAF: Paroxysmal atrial fibrillation;

PMLAGP: Posteromedial left atrial ganglionated plexus;

PLLAGP: Posterolateral left atrial ganglionated plexus; S: Success; SP: Slow pathway; SVT: Supraventricular tachycardia; VA: Ventriculoatrial; Y: Yes.

\section{Competing interests}

The authors declare that they have no competing interests.

\section{Authors' contributions}

SHY, MYC analyzed and interpreted the patient's clinical and laboratory data. $\mathrm{HSH}, \mathrm{MX}$, XJW performed the literature review and wrote the manuscript. RX critically revised the manuscript. All authors have read and approved the final version of this manuscript.

\section{Acknowledgements}

This study was supported by the Independent Innovation foundation for Jinan Science and Technology Development Planning (NO. 201311020) and the Shandong Taishan Scholarship (Suhua Yan).

Received: 25 May 2014 Accepted: 3 June 2014

Published: 10 June 2014

\section{References}

1. Zipes DP, DiMarco JP, Gillette PC: Guidelines for clinical intracardiac electrophysiological and catheter ablation procedures. A report of the American College of Cardiology/American Heart Association Task Force on Practice Guidelines. Circulation 1995, 92:673-691.

2. Joseph JP, Rajappan K: Radiofrequency ablation of cardiac arrhythmias: past, present and future. QJM 2012, 105:303-314.

3. Schlapfer J, Kappenberger L, Fromer M: Bezold-Jarisch-like phenomenon induced by radiofrequency ablation of a left posteroseptal accessory pathway via the coronary sinus. J Cardiovasc Electrophysiol 1996, 7:445-449.

4. Tsai CF, Chen SA, Chiang CE, Tai CT, Lee SH, Wen ZC, Chen YJ, Yu WC, Huang $J L$, Feng AN, Chang MS: Radiofrequency ablation-induced asystole during transaortic approach for a left anterolateral accessory pathway: a Bezold-Jarisch-like phenomenon. J Cardiovasc Electrophysiol 1997, 8:694-699.

5. Duru F, Bauersfeld U, Candinas R: Autonomic effects of radiofrequency catheter ablation. Europace 2000, 2:181-185. 
6. Mathuria N, Bobek J, Afshar H: Sinus arrest during radiofrequency ablation of the atrioventricular-node slow pathway: implications and possible mechanisms. Tex Heart Inst J 2009, 36:477-479.

7. Ulus T, Gorenek B, Nasifov M, Morrad B: Asystole induced by radiofrequency catheter ablation of slow pathway. J Electrocardiol 2013, 46:57-59.

8. Tsai CF, Chen SA, Tai CT, Chiou CW, Prakash VS, Yu WC, Hsieh MH, Ding YA, Chang MS: Bezold-Jarisch-like reflex during radiofrequency ablation of the pulmonary vein tissues in patients with paroxysmal focal atrial fibrillation. J Cardiovasc Electrophysiol 1999, 10:27-35.

9. Efremidis M, Letsas KP, Pappas L, Filippatos G, Sideris A, Kardaras F: Excessive vagal response during left atrial ablation in a patient with paroxysmal atrial fibrillation. Hell J Cardiol 2009, 50:227-229.

10. Capulzini L, Chierchia GB, Sarkozy A, Paparella G, de Asmundis C, Brugada P: Extreme bradycardia during pulmonary vein isolation: keep on ablating in that region. $J$ Cardiovasc Med 2009, 10:267-270.

11. Mark AL: The Bezold-Jarisch reflex revisited: clinical implications of inhibitory reflexes originating in the heart. J Am Coll Cardiol 1983, 1:90-102.

12. Warner HR, Cox A: A mathematical model of heart rate control by sympathetic and vagus efferent information. J Appl Physiol 1962, 17:349-355.

13. Calkins H, Kuck KH, Cappato R, Brugada J, Camm AJ, Chen SA, Crijns HJ, Damiano RJ Jr, Davies DW, DiMarco J, Edgerton J, Ellenbogen K, Ezekowitz MD, Haines DE, Haissaguerre M, Hindricks G, lesaka Y, Jackman W, Jalife J, Jais P, Kalman J, Keane D, Kim YH, Kirchhof P, Klein G, Kottkamp H, Kumagai K, Lindsay BD, Mansour M, Marchlinski FE, et al: 2012 HRS/EHRA/ECAS expert consensus statement on catheter and surgical ablation of atrial fibrillation: recommendations for patient selection, procedural techniques, patient management and follow-up, definitions, endpoints, and research trial design: a report of the Heart Rhythm Society (HRS) Task Force on Catheter and Surgical Ablation of Atrial Fibrillation. Heart Rhythm 2012, 9:632-696.

14. Pokushalov E, Romanov A, Shugayev P, Artyomenko S, Shirokova N, Turov A, Katritsis DG: Selective ganglionated plexi ablation for paroxysmal atrial fibrillation. Heart Rhythm 2009, 6:1257-1264.

15. Po SS, Nakagawa H, Jackman WM: Localization of left atrial ganglionated plexi in patients with atrial fibrillation. J Cardiovasc Electrophysiol 2009, 20(10):1186-1189.

16. Kondo $\mathrm{Y}$, Ueda M, Watanabe M, Ishimura M, Kajiyama T, Hashiguchi N, Kanaeda T, Nakano M, Hiranuma Y, Ishizaka T, Matsumiya G, Kobayashi Y: Identification of left atrial ganglionated plexi by dense epicardial mapping as ablation targets for the treatment of concomitant atrial fibrillation. Pacing Clin Electrophysiol 2013, 36:1336-1341.

17. Oh S, Zhang Y, Bibevski S, Marrouche NF, Natale A, Mazgalev TN: Vagal denervation and atrial fibrillation inducibility: epicardial fat pad ablation does not have long-term effects. Heart Rhythm 2006, 3:701-708.

\section{doi:10.1186/1756-0500-7-35}

Cite this article as: Hu et al.: Sudden asystole during radiofrequency ablation: a case report and literature review. BMC Research Notes 2014 7:351.

\section{Submit your next manuscript to BioMed Central and take full advantage of:}

- Convenient online submission

- Thorough peer review

- No space constraints or color figure charges

- Immediate publication on acceptance

- Inclusion in PubMed, CAS, Scopus and Google Scholar

- Research which is freely available for redistribution 